\title{
Satisfaction with the performance of emergency medical services: a case study in Tabriz
}

\author{
Fereshteh Jamali' ${ }^{1}$, Haniyeh Ebrahimibakhtavar' ${ }^{2}$, Mahbubeh Zomordi Torkdari², Farzad Rahmani2 ${ }^{*}$ \\ 'Students' Research Committee, Tabriz University of Medical Sciences, Tabriz, Iran \\ 2Emergency Medicine Research Team, Tabriz University of Medical Sciences, Tabriz, Iran
}

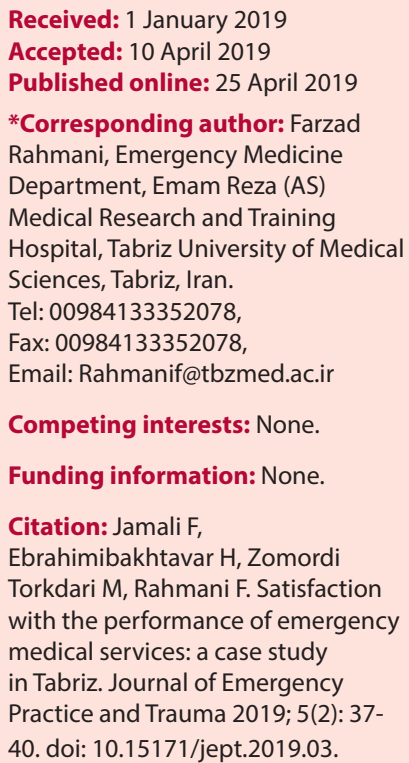

\begin{abstract}
Objective: Assessing patients' satisfaction with emergency medical services (EMSs) is an important managerial point of view. The present study aimed to assess the patients' satisfaction with EMS in Tabriz, Iran.

Methods: This is a descriptive-analytic study that was done in the prehospital emergency and disaster management center of Tabriz University of Medical Sciences. In this study, from May to December 2017, we included 659 patients who were transferred to hospitals with our prehospital emergency services. A valid and reliable questionnaire was used to evaluate the satisfaction of patients about the performance of prehospital EMSs.

Results: Data of 659 patients were evaluated. Trauma was the most common cause of contact with 115 and help request (33.83\%). The results indicated that patients' level of satisfaction was good and very good. The highest level of satisfaction was related to the treatment of patients and use of medical equipment in the ambulance $(n=578$ patients, $87.7 \%)$ as well as the treatment practiced by the emergency medical technicians (EMTs) ( $n=575$ patients, $87.24 \%$ ). However, the lowest level of satisfaction was related to the absence of an active EMT in the rear cabin when transferring patients to the hospital $(n=337$ patients, $51.14 \%$ ) and transfer of the patient from the accident scene to the ambulance ( $n=410$ patients, $62.21 \%$ ).

Conclusion: Patients' satisfaction with EMS was optimal. The minimum and maximum satisfaction rates were related to the absence of an active EMT in the rear cabin, treatment of the patient, and use of medical equipment.

Keywords: Ambulance, Prehospital emergency care, Emergency medical services, Satisfaction
\end{abstract}

\section{Introduction}

Emergency Medical Service (EMS) refers to a set of emergency medical cares which involves emergency treatment or transfer of the injured patient outside the hospital setting (1). Patient satisfaction is an important indicator for the quality of healthcare services (2). Indeed, paying close attention to patients' point of view can fundamentally enhance the quality of the healthcare services provided in all sections of the healthcare system. Patient satisfaction is about patients' experiences concerning the healthcare system which must be taken into account and continuously measured while planning for services and facilities (3).

In recent years, it has been increasingly important to assess the quality of healthcare services from the patients' point of view, since it is the patients' right to express their ideas on the services they receive (4). The reason is that EMS is generally the first department in healthcare systems which primarily deals with critically-ill and emergency patients (5). EMS is an essential element in the treatment of emergency patients in prehospital settings as well as their transfer to the hospitals in the urban healthcare system (6).

Emergency medical centers are considered to be the most important pillar of healthcare provision worldwide, and the main goal of this system is to provide satisfactory services within the shortest possible time and in line with the scientific standards (7). The more appropriate, accurate, and faster the services become, the lower the number of deaths and mortality rate. In this regard, people's confidence to EMS will be increased (8). Experts believe that the level of satisfaction with the services is a quality measurement indicator, and improving this quality requires the proper knowledge of the current situations and investigation of the problems in this regard (9).

The present study aimed to assess the level of patients' satisfaction receiving EMS in Tabriz in 2017. In this regard, it is possible to enhance the level of efficacy in 
such departments as well as patients' satisfaction by examining the strategies in order to develop and improve the emergency system.

\section{Methods}

This descriptive analytic study was conducted on patients who contacted the Emergency Department (ED) 115 of Tabriz during summer and fall of 2017 and were then transferred to an ED or underwent outpatient treatment at home.

Inclusion criteria included patients who were in need of urgent measures and those who contacted the medical emergency center. Exclusion criteria included phone contacts beyond the study time, failure of patients or their companions to contact the quality control expert, and unwillingness to participate in the study.

In order to collect data, the Satisfaction Questionnaire from Disaster and Emergency Medical Management Center of East Azerbaijan was used. This questionnaire included questions concerning patients' demographic characteristics and their level of satisfaction with different parts of this organization. The content validity of the questionnaire was obtained by receiving the opinions of some members of the Faculty of Emergency Medicine of Tabriz University of Medical Sciences. In this regard, some necessary changes were made to the questionnaire based on their opinions. In order to evaluate the reliability of the questionnaire, we used Cronbach's alpha test. After collecting thirty checklists, the questionnaires were analyzed and Cronbach's alpha was 0.8 . In this study, 3-5 patients per day that were transferred to hospitals with ambulances were selected and the trained expert working in the quality control unit of our center made phone contacts with the patients or their families to complete the questionnaire.

The above checklist consisted of two parts. The first part included demographic information of patients including, age, gender, date of contact, type of disease, etc. The second part included 9 questions on patients' satisfaction with the EMS encompassing questions on how the dispatcher treated the patients, obtaining the patient's medical history, the time taken for the ambulance to arrive by the patient's bedside, how emergency medical technicians (EMTs) treated the patient, use of equipment, patient treatment, procedure of transferring the patient in the ambulance, presence of the EMT in the rear cabin during the transfer process, the appearance and cleanliness status of ambulances, and the procedure adopted for transferring the patient to the ED of the hospital. These questions were answered based on a 5-point Likert Scale ranging from completely satisfied (5), satisfied (4), have no idea (3), dissatisfied (2), and completely dissatisfied (1).

All analyses were conducted using SPSS version 17. Data description was performed using descriptive statistics (frequency, percentage, mean, and standard deviation). Normal distribution was assessed using Kolmogorov-
Smirnov test. Spearman correlation test was employed to investigate the correlation between the data. $P$ value less than 0.05 was considered as the significant level.

\section{Results}

In this study, the data of 659 patients who contacted the communication center of the medical emergency of Tabriz were evaluated, of whom 381 (57.8\%) were males. The mean age of patients was $48.17 \pm 21.92$ years. Results revealed that 41 patients $(6.22 \%)$ were below 20, 239 patients $(36.3 \%)$ were between 21 and 40,180 patients (27.3\%) were between 41 and 60, 147 patients $(22.3 \%)$ were between 61 and 80 , and 52 patients $(7.9 \%)$ were above 80 years of age.

There were also questions on the type of disease and initial complaints of patients who had contacted the EMS. Results demonstrated that trauma ( $\mathrm{n}=233$ cases, $33.83 \%$ ) and panic attack ( $\mathrm{n}=18$ cases, $2.73 \%$ ) were the most and least common causes of making phone contact with the EMS of Tabriz, respectively.

In this study, 154 patients (23.4\%) were treated at home or were not willing to be transferred to the hospital, and the most common cause of non-transfer was the improvement of patients at home ( $\mathrm{n}=116$ cases). Also, other reasons for not transferring patients to the hospitals included the lack of patient cooperation ( $\mathrm{n}=13$ cases), lack of cooperation of their companions ( $\mathrm{n}=11$ cases), aging ( $\mathrm{n}=5$ cases), patient transfer by companions ( $\mathrm{n}=3$ cases), and other causes ( $\mathrm{n}=6$ cases).

Table 1 shows the results on patients' and their companions' satisfaction with the quality of services provided by the EMS in the County of Tabriz. As this table indicates, the highest satisfaction was related to the use of medical equipment and treatment of patients $(\mathrm{N}=578$ patients, $87.7 \%$ ), while the minimum satisfaction rate was for the absence of an active EMT in the rear cabin during the transfer process ( $\mathrm{N}=337$ patients, $51.14 \%$ ).

Also, we evaluated the association between patients' gender and chief complaints with their satisfaction rate. We did not observe any statistical significant relationship between gender as well as the main complaint of these patients with their level of satisfaction $(P$ value $>0.05)$.

\section{Discussion}

The present study evaluated the patients' satisfaction with the quality of EMS in Tabriz in 2017. In this study, the maximum level of satisfaction was related to the use of medical equipment and treatment of the patients, while the minimum satisfaction was observed for the absence of an active EMT in the rear cabin during the transfer to the hospital.

Patients' satisfaction with the services provided to them is one of the criteria for assessing the quality of services provided by healthcare systems (2). Many factors contribute to the successful delivery of high-quality healthcare services in EMS. Among these factors, the presence 
Table 1. Patients or their families' opinions about prehospital emergency system

\begin{tabular}{|c|c|c|c|c|c|}
\hline Variables & Very good & Good & Moderate & Poor & Very poor \\
\hline 1. Interaction and communication of Emergency Medical Dispatcher & $38(5.76 \%)$ & $522(79.21 \%)$ & $62(9.4 \%)$ & $27(4.1 \%)$ & $10(1.5 \%)$ \\
\hline 2. Taking history by the Emergency Medical Dispatcher & $32(4.85 \%)$ & $536(81.33 \%)$ & $62(9.40 \%)$ & $19(2.88 \%)$ & $10(1.5 \%)$ \\
\hline 3. Ambulance arrival time on the patient's bedside & $25(3.79 \%)$ & $450(68.3 \%)$ & $89(13.5 \%)$ & $61(9.25 \%)$ & $34(5.16 \%)$ \\
\hline 4. Interaction and communication of EMT & $17(2.57 \%)$ & $558(84.67 \%)$ & $56(8.49 \%)$ & $17(2.58 \%)$ & $11(1.67 \%)$ \\
\hline $\begin{array}{l}\text { 5. How to approach to the patients and the use of medical equipment } \\
\text { by EMT }\end{array}$ & $18(2.73 \%)$ & $560(84.97 \%)$ & 39 (5.91\%) & 27 (4.09\%) & $15(2.27 \%)$ \\
\hline 6. How to transfer the patients into the ambulance by EMT & $98(14.87 \%)$ & $312(47.34 \%)$ & $100(15.18 \%)$ & $74(11.23 \%)$ & $75(11.38 \%)$ \\
\hline $\begin{array}{l}\text { 7. Active presence of EMT in the cabin of ambulance during the transfer } \\
\text { of the patient to the hospital }\end{array}$ & $53(8.04 \%)$ & $284(43.10 \%)$ & $152(23.07 \%)$ & $115(17.45 \%)$ & $55(8.34 \%)$ \\
\hline 8. Status of ambulance cleaning & $258(39.15 \%)$ & $231(35.05 \%)$ & $132(20.03 \%)$ & $38(5.77 \%)$ & $0(0.0 \%)$ \\
\hline $\begin{array}{l}\text { 9. How to transfer the patients to the emergency ward of the hospital } \\
\text { by EMT }\end{array}$ & $206(31.26 \%)$ & $255(38.69 \%)$ & $81(12.29 \%)$ & $61(9.25 \%)$ & $56(8.50 \%)$ \\
\hline
\end{tabular}

of skilled personnel, speed of delivery of services, and presence of vehicles equipped with appropriate medical equipment are of particular importance. Today, EMSs play a significant role in providing healthcare services to patients. A contentment of the services provided by EMS can have a significant impact on the total satisfaction of patients about healthcare systems (10).

Nowadays, a growing importance is being attached to ambulances for the timely transfer of patients to medical centers as the availability of equipped ambulances in emergency situations can play the most important role in saving the patients' life and reducing the subsequent casualties (11).

Kuisma et al suggested that patients' dissatisfaction increases when they are not transferred to their desired hospital. Also, the high inefficiency in management of patients' problems and lack of proper communication with patients by EMS personnel are two other factors affecting patients' satisfaction with EMS (12). Swain et al also found that unnecessary transfer is another factor that increases patients' dissatisfaction. Accordingly, in some countries, including New Zealand, the pre-hospital emergency has made its efforts to treat the patients requiring services at home as much as possible and avoid transferring patients to the crowded wards of the EDs of the hospitals (6). A study conducted by Bahrami et al in Yazd also showed that more than $70 \%$ of patients were satisfied with the emergency performance. In this study, the highest and lowest satisfaction rates were related to the efficiency of EDs and technicians' performance, respectively (13). Mehrabian et al reported in a study that there was no significant difference between the satisfaction with EMSs based on age, a history of using EMSs, mission time, and dependence on the healthcare system (14).

The results of the present research indicated that the majority of patients included in our study had optimal satisfaction about the healthcare services provided by EMS. One of the reasons justifying the high satisfaction level in the present study is the free EMSs, which is consistent with some other studies showing a significant relationship between the treatment cost and patient satisfaction $(15,16)$. There was no statistical significant relationship between the satisfaction level of patients and gender as well as their main complaint. In this regard, Malekmakan et al (17) and Jafari et al (18) showed no significant difference in the satisfaction level of the two genders, which is consistent with the present study. However, Woods and Heidari showed that gender does not have a role in patient satisfaction (19). Another issue that affects the satisfaction of patients with EMSs is the condition of ambulances and technicians, which is observed and judged by patients (20).

\section{Conclusion}

The results of the present study suggested that patients had optimal satisfaction with EMSs. In this study, the maximum level of satisfaction was related to the use of medical equipment and treatment of the patients, while the minimum satisfaction was observed for the absence of an active EMT in the rear cabin during the transfer to the hospital. Hence, measures are required to meet patients' expectations. Considering the role of the six factors in patient satisfaction, 1) training communication skills for nurses, physicians, and EMTs, 2) designing and strengthening a customer relationship unit (including patient, companion, and other people of the society), 3) setting up follow-up systems during the waiting time, 4) holding specialized trainings for operators, 5) recruiting at least one physician in message centers, and 6) adhering to the compliance plan are among the factors which can lead to improved patient satisfaction.

\section{Limitations}

This study had some limitations. We did not have access to the telephone numbers of the statistical population. Also, some people (240 unsuccessful calls) refused to answer questions because of distrust or lack of time. The present study did not investigate patients' level of education and place of residence. Therefore, the goals associated with these variables were not evaluated. Other limitations 
included the fact that patients or their companions did not respond properly to the expert's questions and provided wrong phone numbers in the medical records.

\section{Ethical issues}

This study was approved by the Ethics Committee of Tabriz University of Medical Sciences with the code number IR.TBZMED.REC.1396.796. Patients' confidentiality was protected by not mentioning the names of the participants when reporting the results.

\section{Authors contributions}

All authors read and approved the manuscript. FJ, $\mathrm{HEB}$, and $\mathrm{MZ}$ performed the data collection, writing, critical revision and drafting the manuscript. FR and HEB undertook the major parts of the study design and performed the statistical analysis, data analysis, and data interpretation.

\section{Acknowledgements}

The authors are grateful to all the personnel of disaster and emergency management center of Eastern Azerbaijan Province. This article was written based on a dataset of MD thesis registered in Tabriz University of Medical Sciences. [Number: 59116, Approved date: 01.03.2017].

\section{References}

1. Reid BO, Rehn M, Uleberg O, Pleym LEN, Kruger AJ. Interdisciplinary cooperation in a physician-staffed emergency medical system. Acta Anaesthesiol Scand 2018; 62(7): 1007-13. doi: 10.1111/aas.13112.

2. Eshghi M, Rahmani F, Derakhti B, Robai N, Abdollahi F, Tajoddini S. Patient satisfaction in the emergency department: a case of Sina hospital in Tabriz. J Emerg Pract Trauma 2016; 2(1): 16-20. doi: 10.15171/jept.2015.06.

3. Entezari-asl M, Motamedi F. Degree of satisfaction among patients referring to the emergency rooms of hospital of Ardabil University of Medical Sciences, 2000. Journal of Ardabil University of Medical Sciences 2003; 3(2): 20-7. [In Persian].

4. Bailey ED, Sweeney T, National Association of EMS Physicians. Considerations in establishing emergency medical services response time goals. Prehosp Emerg Care 2003; 7(3): 397-9.

5. Grogan S, Conner M, Norman P, Willits D, Porter I. Validation of a questionnaire measuring patient satisfaction with general practitioner services. Qual Health Care 2000; 9(4): 210-5. doi: 10.1136/qhc.9.4.210

6. Swain AH, Al-Salami M, Hoyle SR, Larsen PD. Patient satisfaction and outcome using emergency care practitioners in New Zealand. Emerg Med Australas 2012; 24(2): 175-80. doi: 10.1111/j.1742-6723.2011.01525.x.

7. Saberi Nia A, Nekouei Moghadam M, Mahmoudi Meymand F. Identify stressful factors causing dissatisfaction in pre-hospital emergency personnel in Kerman. Payavard Salamat 2013; 6(6): 489-97. [In Persian].

8. Bidari A, Abbasi S, Farsi D, Saeidi H, Mofidi M, Radmehr $\mathrm{M}$, et al. Quality assessment of prehospital care service in patients transported to Hazrat-e-Rasoul akram hospital. Medical Journal of Tabriz University of Medical Sciences 2007; 29(3): 43-6. [In Persian].

9. McKinley R, Roberts C. Patient satisfaction with out of hours primary medical care. Qual Health Care 2001; 10(1): 23-8. doi: 10.1136/qhc.10.1.23

10. Jaklic TK, Kovac J, Maletic M, Bunc KT. Analysis of patient satisfaction with emergency medical services. Open Med (Wars) 2018; 13: 493-502. doi: 10.1515/med-2018-0073.

11. Bahadori M, Ghardashi F, Izadi AR, Ravangard R, Mirhashemi S, Hosseini SM. Pre-hospital emergency in Iran: A systematic review. Trauma Mon 2016; 21(2): e31382. doi: 10.5812/traumamon.31382.

12. Kuisma M, Maatta T, Hakala T, Sivula T, Nousila-Wiik M. Customer satisfaction measurement in emergency medical services. Acad Emerg Med 2003; 10(7): 812-5.

13. Bahrami MA, Ranjbar Ezzatabadi M, Maleki A, Asqari R, Ahmadi Tehrani GH. A survey on the yazd pre-hospital emergency medical services' performance assessment, 2009-2010. Toloo-e-Behdasht 2011; 9(4): 45-58. [In Persian].

14. Mehrabian F, Faize Sabet A, Ezatpana Abatari L. Patients satisfaction of pre-hospital emergency services in Rasht. Journal of Guilan University of Medical Sciences 2017; 26(103): 30-6. [In Persian].

15. Heydari H, Kamran A, Zali ME, Novinmehr N, Safari M. Customers' satisfaction about prehospital emergency medical services in Lorestan, Iran. Electron Physician 2017; 9(3): 3974-9. doi: 10.19082/3974.

16. Price L. Treating the clock and not the patient: ambulance response times and risk. Qual Saf Health Care 2006; 15(2): 127-30. doi: 10.1136/qshc.2005.015651.

17. Malekmakan L, Haghpanah S, Moravej H, Sharifi M. The Effect of intervention on patient's satisfaction in emergency departments of the hospitals affiliated to Shiraz university of medical sciences. Journal of Jahrom University of Medical Sciences 2010; 7(3): 52-61. [In Persian].

18. Jafari F, Zayeri F, Johari Z. The satisfaction of those who recourse to the to the health centers of Shahid Beheshti university of medical sciences. Daneshvar 2007; 4(66): 1522. [In Persian].

19. Woods SE, Heidari Z. The influence of gender on patient satisfaction. J Gend Specif Med 2003; 6(4): 30-5.

20. Williamson $\mathrm{K}$, Ramesh $\mathrm{R}$, Grabinsky A. Advances in prehospital trauma care. Int J Crit Illn Inj Sci 2011; 1(1): 44-50. doi: 10.4103/2229-5151.79281. 